\title{
Neurological, cardiovascular and metabolic effects of mefloquine in healthy volunteers: a double-blind, placebo-controlled trial
}

\author{
TIMOTHY M. E. DAVIS, LAWRENCE G. DEMBO, SALLY A. KAYE-EDDIE, BENJAMIN J. HEWITT, \\ ROBERT G. HISLOP \& KEVIN T. BATTY \\ University of Western Australia, Department of Medicine, Fremantle Hospital, Fremantle, Western Australia
}

1 To assess neurological, cardiovascular, metabolic and other side-effects of mefloquine given in conventional prophylactic dose to healthy volunteers, a double-blind, randomized, placebo-controlled trial was conducted. In addition, the identity of the active drug was concealed until the end of the trial.

2 A total of 106 healthy adults were recruited, of whom 95 (mean age 24 years; $45 \%$ males) completed the full study protocol.

3 Subjects had a baseline assessment, received placebo as first dose, were randomized to mefloquine $250 \mathrm{mg}$ or placebo weekly for 4 weeks starting a week later, and were reassessed after the 2nd and 4th active/placebo doses. Subjects kept a daily symptom diary from 2 weeks before until 2 weeks after the dosing period.

4 Plasma mefloquine assay suggested compliance in all 46 subjects allocated active treatment (week 5 mean \pm s.d.; $2.35 \pm 0.94 \mu \mathrm{mol}^{-1}$ ). Mefloquine did not alter calcium homoeostasis but produced a mean $0.5 \mathrm{mmol} 1^{-1}$ fall in serum glucose over the study period $(P<0.001)$ and relative hyperinsulinaemia. Symbol digit modalities, and digit forwards and backwards test scores, were similar in active and placebo groups across the three assessments, as were lying/standing blood pressure and high-tone hearing loss. Electrocardiographic $\mathrm{QT}_{\mathrm{c}}$ interval prolongation and diarrhoea were mild but transient side-effects of mefloquine $(P<0.01)$. Neurological symptoms were comparable in the two groups throughout the study. There was no evidence of drug toxicity in 11 subjects who withdrew.

5 Mefloquine prophylaxis does not appear to produce low-grade but debilitating neurological symptoms or to alter the results of sensitive tests of cerebral function. However, there may be situations in which mefloquine might contribute to hypoglycaemia and cardiac dysrhythmias.

Keywords mefloquine side-effects neurological function glucose insulin

\section{Introduction}

Mefloquine can be used as prophylaxis or treatment for malaria and covers most multi-drug resistant strains of Plasmodium falciparum. Although large-scale studies have affirmed its relative safety and efficacy as a prophylactic agent [1-3], serious side-effects including isolated cutaneous [4-6] and marrow toxicity $[7,8]$ have been reported. Severe neuropsychiatric reactions are more common [9], with an estimated frequency of 1 in 10,000 to 14,000 prophylactic courses $[10,11]$. Review of 59 such reactions has revealed that a large proportion of patients had a past history of neurological or psychiatric diseases such as epilepsy and manicdepressive illness [12].

Milder and more common neurological effects including dizziness, vertigo, paraesthesiae, depression, fatigue, insomnia, and poor concentration and memory can 
occur soon after ingestion of a single mefloquine dose and persist for weeks afterwards [1, 13-17]. However, physician warnings of neuropsychiatric reactions, and the stresses and stimulation of travel, might produce such symptoms in susceptible individuals. A review of 109 trials involving healthy subjects has revealed an adverse event frequency on placebo of $19 \%$ in single and $28 \%$ in multiple dose studies [18]. Headache, drowsiness, poor concentration and aesthenia are reported commonly in young volunteers.

To investigate whether mild but debilitating neurological symptoms occur during a conventional prophylactic mefloquine regimen and, if so, whether metabolic disturbances might contribute, we conducted a doubleblind, randomized placebo-controlled study in healthy volunteers who were unaware of the identity of the drug under evaluation until the end of the trial.

\section{Methods}

\section{Subjects}

Subjects were healthy adult staff and students at teaching hospitals in Perth, Western Australia. All completed a standard medical questionnaire. Those with a past history of psychiatric conditions, or neurological, cardiac, hepatic or renal disease were excluded, as were pregnant or breast-feeding females and those with a known allergy to, or taking medication known to interact with, quinoline drugs. None of the subjects had taken mefloquine in the 3 months before study.

\section{Methods}

The study protocol was approved by the University of Western Australia Human Rights Committee. Witnessed informed consent was obtained after full verbal and written explanations of procedures, including advice that the identity of the active formulation would be concealed until the end of the study unless an adverse event occurred. Tablets were prepared in individually numbered but otherwise unlabelled containers. Allocation of active or placebo formulation was by a random number code generated by independent Fremantle Hospital Pharmacy staff who kept the code strictly confidential until after the last volunteer had completed the protocol. Subjects were informed of procedures to be followed in the event of an adverse reaction and an independent trial monitor was available in case the randomisation code needed to be broken. Pregnancy tests were offered to all females who were advised to ensure adequate contraception during, and for 3 months after, the dosing period. A diary containing a list of commonly reported side-effects [2, 3] (see below), together with spaces for other symptoms if required, was issued. Subjects were asked to rate each standard and self-entered symptom daily at the same time on a scale of 0 (absent) to 9 (severe).

Subjects reattended after 1 week of symptom recording to have the diary checked for completeness and to undergo standard tests. The first tablet, placebo in all cases, was given under supervision. Subjects received four mefloquine (Larium ${ }^{\circledR}$, Hoffman La Roche, Basel, Switzerland) $250 \mathrm{mg}$ or identical placebo tablets to be taken weekly, starting a week after the initial placebo. Venous blood was drawn for measurement of plasma mefloquine and its carboxy metabolite, serum glucose, insulin, ionised calcium, phosphate, magnesium and albumin concentrations. The symbol digit modalities test (SDMT) [19] and digit span forwards and backwards tests [20] were administered. An electrocardiogram (ECG) was taken, blood pressure was taken supine and after two minutes' standing using an automated system (Dynamap 1846 SX, Critikon Inc., Florida, USA), and hearing loss at $6 \mathrm{kHz}$ was measured in $5 \mathrm{~dB}$ steps using a pre-calibrated audiometer (MA40, Maico Hearing Instruments, Minneapolis, USA).

Subjects were asked to reattend for assessment 1 day after the third and fifth (last) tablets had been taken, at a time when plasma mefloquine concentrations would be expected to be close to maximal post-dose. At these visits, diaries were reinspected and compliance checked by questioning and tablet count. Blood sampling and all other tests were repeated. Each subject was asked to maintain the diary until 2 weeks after the last assessment when it was collected. Assay of mefloquine and metabolite was by high performance liquid chromatography [21]. Serum glucose, albumin, phosphate and magnesium concentrations were analysed using Chem-1 methods (Bayer Diagnostics, Tarrytown, NY). Serum ionised calcium was determined using an ICA 2 analyser (Radiometer, Copenhagen) and corrected to $\mathrm{pH}$ 7.40. Serum insulin was measured immunoenzymometrically (Tosoh, Tokyo, Japan).

\section{Data analysis}

To detect at least a $10 \%$ difference between the two groups for both SDMT [19] and digit span forwards test [20] using a 0.05 significance level at $80 \%$ power, a minimum sample size of 47 in each group was required. For diary data, a positive symptom was taken as a rating $\geq 1$ for at least 1 day during a given week. Major symptoms were those rated $\geq 5$ on any one day of the week. To assess the effect of mefloquine on symptoms, the proportions of subjects in whom a symptom rating worsened during the first week after active drug or unsupervised placebo were calculated using the rating from the week following the initial supervised and universal placebo as baseline. The relative risk was taken as the ratio of the proportion worsening after mefloquine to that after unsupervised placebo. This analysis was repeated for the week after the last dose, again using the rating after supervised placebo as baseline. For psychometric tests, retest gain (the percentage improvement or deterioration in scores relative to baseline) at the second and third assessments was calculated by the formula:

score at follow-up-score at initial assessment score at initial assessment 
Serum insulin concentrations were log-transformed before analysis. Statistical analysis was by parametric methods using SPSS for Windows (SPSS Inc., Chicago, USA). Two-sample comparisons were by Student's $t$-test and multiple comparisons by analysis of variance. Pearson's correlation co-efficient was used to assess associations between variables.

\section{Results}

Subjects

Of 106 randomized volunteers, $95(90 \%)$ completed all study procedures. Forty-six of these received mefloquine and 49 placebo. Age and sex distributions of those on mefloquine $(23.5 \pm 6.3$ years, $51 \%$ males $)$ were not significantly different from those on placebo $(24.6 \pm 7.8$ years, $39 \%$ males; $P>0.1)$, and body weights were similar $(65.3 \pm 11.2$ and $66.7 \pm 10.6 \mathrm{~kg}$ respectively, $P=$ 0.26). Eight subjects withdrew after initial assessment and three after the second. Follow-up of these individuals revealed no toxicity in those allocated mefloquine. Reasons for withdrawal were a lack of time to devote to study procedures, including completion of diaries, in 10 individuals and accidental injury in a volunteer receiving placebo.

\section{Compliance and plasma mefloquine concentrations}

Compliance appeared full on direct questioning and tablet count at follow-up visits though several volunteers reported taking tablets a day or two late between assessments. Plasma mefloquine and its carboxy metabolite were measurable at both the second and third assessments in all allocated active formulation. After two doses, plasma mefloquine ranged from 0.25 to 2.82 (mean 1.52) $\mu \mathrm{mol} 1^{-1}$ and carboxy-metabolite concentrations were from 0.25 to 6.24 (mean 2.35$) \mu \mathrm{mol} 1^{-1}$; the average metabolite: drug ratio was 2.0. After the last dose, the equivalent figures were 0.94 to 5.37 (mean 2.35) $\mu \mathrm{mol} 1^{-1}$ and 1.4 to 9.5 (mean 5.3) $\mu \mathrm{mol} 1^{-1}$ respectively, with an average carboxy-metabolite: mefloquine ratio of 2.6.

\section{Symptom reporting}

The most common symptom during the run-in week and week after universal placebo was headache. Approximately half the total sample experienced at least mild headache (rating $\geq 1$ ) on one or more days during this period (see Table 1). This proportion remained relatively constant during the ensuing six weeks. There was no increase in the relative risk of this symptom occurring after the first and last mefloquine dose (see Table 1). The number of subjects experiencing major headache (rating $\geq 5$ ) remained low (1 or 2 participants) and comparable between the two groups throughout.
A similar pattern was seen for the listed diary symptoms lethargy, cough and the other less frequently positive $(<10 \%)$ non-gastrointestinal complaints skin rash, itch, dizziness, hearing loss, tinnitus, memory loss, visual disturbance, insomnia and vivid dreams. In the first week after mefloquine administration, mild abdominal pain and nausea increased but this trend did not reach statistical significance (see Table 1). However, diarrhoea worsened significantly after mefloquine (see Table $1 ; P<0.01$ ), an effect which was transient and not associated with an increase in its rating as a major symptom (see Figure 1).

\section{Biochemical variables}

Serum ionised calcium concentrations remained stable over the study period (mean \pm s.d.; $1.24 \pm 0.03 \mathrm{mmol}^{-1}$ at first and final assessment for subjects on active and placebo treatment respectively), as did serum magnesium and phosphate $(P>0.5)$. The mean serum albumin fell approximately $1 \mathrm{~g} \mathrm{l}^{-1}$ in both groups $(P<0.004$ baseline $v s$ final assessment). A progressive fall in serum glucose over the three assessments was observed in those on active formulation $\left(5.0 \pm 0.7\right.$ vs $4.5 \pm 0.8 \mathrm{mmol}^{-1}$ at baseline and final assessments respectively; $P=0.0005)$. This fall was proportionately greater than that of serum albumin and was accompanied by a significant increase in serum insulin (geometric mean $[ \pm 1$ s.d. range $] ; 10.6$ [5.7-19.8] vs 15.7 [7.7-31.9] mu $1^{-1}$ respectively; $P=$ 0.01 ; see Figure 2). No significant changes in serum glucose or insulin were seen in the placebo group $(P>0.2$; see Figure 2). Plasma mefloquine concentrations at the final assessment and the change in serum insulin over the 5 weeks of the study did not correlate significantly $(r=0.203 ; \quad P=0.09)$. The lowest serum glucose $\left(2.5 \mathrm{mmol}^{-1}\right)$ occurred in asymptomatic individuals from both groups at the second assessment.

\section{Psychometric testing}

Subjects in the two groups had comparable mean baseline scores for all three tests $(P>0.14)$. There was a significant improvement in retest gain in SDMT and digit span backwards scores during the study in the placebo group $(P<0.01)$. In those allocated mefloquine, significant learning was evident only in the case of SDMT score $(P<0.001)$. However, retest gain at the third assessment was not significantly different between the two groups for DMST $(P=0.25)$, digit forwards $(P=0.16)$ and digit backwards $(P=0.08)$ tests (see Figures 3 and 4).

\section{Audiometric, blood pressure and electrocardiographic changes}

There was no significant hearing loss at $6 \mathrm{kHz}$ in either the active or placebo groups over the three assessments $(P>0.2$; see Table 2$)$. Supine and erect blood pressure also showed no changes during the study $(P>0.2$; see 
Table 1 Percentage of subjects reporting symptoms of at least mild severity (rating $\geq 1$ ) in the week after initial universal placebo (baseline). The relative risk and ( $95 \%$ confidence intervals) of each symptom worsening from baseline after active $(n=46)$ compared with placebo $(n=49)$ treatment is shown for both first and last doses. Other symptoms occured in $<10 \%$ of subjects in both groups.

\begin{tabular}{lllll}
\hline & $\begin{array}{l}\text { Week after placebo } \\
\text { dose (all subjects) }\end{array}$ & & & $\begin{array}{l}\text { Relative risk of symptom worsening in mefloquine } \\
\text { compared with placebo group }\end{array}$ \\
\cline { 2 - 3 } Mefloquine & Placebo & & Week after first dose & Week after last dose \\
\hline Headache & $45 \%$ & $60 \%$ & $1.2(0.5-2.9)$ & $1.3(0.5-3.3)$ \\
Lethargy & $49 \%$ & $43 \%$ & $1.0(0.4-2.5)$ & $1.0(0.5-2.4)$ \\
Abdominal pain & $26 \%$ & $20 \%$ & $2.2(0.7-7.1)$ & $1.1(0.3-3.4)$ \\
Diarrhoea & $27 \%$ & $16 \%$ & $7.6(1.6-36.5)^{*}$ & $2.8(0.8-9.9)$ \\
Cough & $27 \%$ & $14 \%$ & $0.4(0.1-2.2)$ & $1.3(0.5-3.5)$ \\
Nausea & $13 \%$ & $17 \%$ & $2.4(0.7-8.7)$ & $2.2(0.7-7.1)$ \\
\hline
\end{tabular}

$* P<0.01$

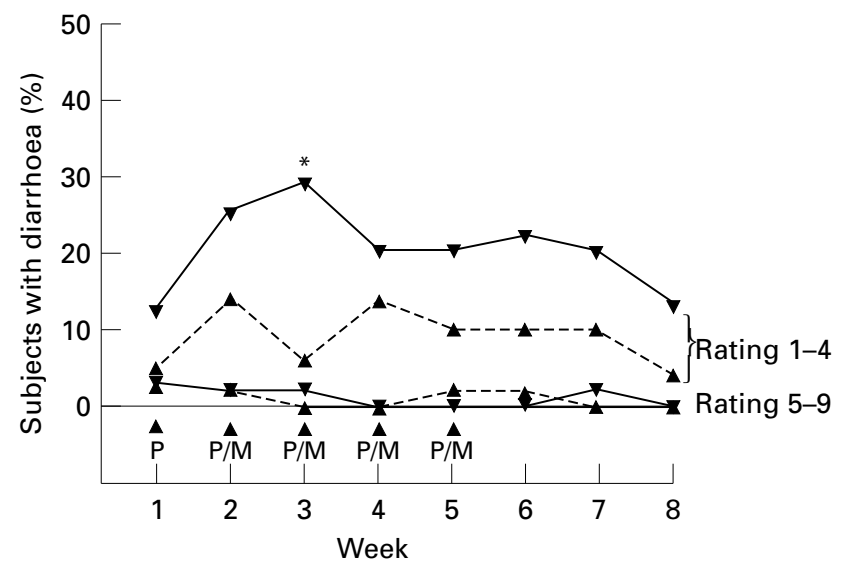

Figure 1 Percentage of patients with mild (rating between 1 and 4 on at least one day of the week; upper two lines) and severe (rating $\geq 5$; lower two lines) diarrhoea in the mefloquine $(\boldsymbol{\nabla}-\boldsymbol{\nabla})$ and placebo $(\boldsymbol{\Delta}---\boldsymbol{\Delta})$ groups. There was a worsening of mild diarrhoea after the first (universal) placebo $(\mathrm{P})$ dose with a further significant increase after the first dose $(\mathrm{P} / \mathrm{M})$ of mefloquine compared to placebo $\left({ }^{*} P<0.01\right)$.

Table 2), as did pulse rate (data not shown). There was a statistically significant prolongation in the electrocardiographic $\mathrm{QT}_{\mathrm{c}}$ interval between the first and second assessments in the subjects who received mefloquine $(P=0.007)$; a less pronounced and later trend was seen in the placebo group $(P=0.03$; see Table 2$)$. Other electrocardiographic indices (including PR interval and QRS duration) were comparable over the three assessments in both groups of subjects (data not shown).

\section{Discussion}

The present study attempted to minimise bias through several strategies. In addition to a double-blind, placebocontrolled design, subjects received an initial placebo before starting regular active medication or identical placebo. The identity of the active formulation was concealed until the end of the trial. Compliance appeared close to complete as assessed from questioning, tablet count and, in those taking mefloquine, by sequential plasma drug and metabolite concentrations. Although strict procedures for monitoring adverse events were in place, the randomization code did not need to be broken prematurely. Subjects who withdrew were assessed subsequently for evidence of side-effects. As a result, a range of objective data was obtained from a sample of healthy young adults typical of travellers to countries in which malaria transmission occurs.

The limitations of the study relate to its environment and duration. Our volunteers remained in familiar home and work surroundings, in contrast to the stimulating world of the traveller. Nevertheless, whether environment accentuates possible mild neuropsychiatric effects of mefloquine is unknown. Although active treatment was given for only 4 weeks, a subject on this regimen is usually considered safe to travel to an area of malaria transmission midway through this period. In addition, side-effects of mefloquine, including those of most interest in the present study, often appear soon after the first dose [1, 13-17]. Steady-state concentrations of mefloquine take 8-10 weeks to achieve [22] at which time concentrations of the carboxylic acid metabolite are, on average, more than twice those of mefloquine [23]. Although the final blood sample was not taken at trough plasma concentrations in our subjects, metabolite concentrations were usually more than double those of mefloquine itself. In addition, final peak plasma mefloquine concentrations in more than one in seven of our subjects were within the range for healthy young travellers $\left(>3.3 \mu \mathrm{mol} \mathrm{l}^{-1}\right)$ found at the end of 13 weeks of mefloquine $250 \mathrm{mg}$ weekly [22].

At each assessment, subjects were reminded that any symptoms should be rated daily. This encouragement might have contributed to the high rate of self-reported headache, lethargy, abdominal pain, diarrhoea, cough and nausea. Nevertheless, mild and transient diarrhoea was the only symptom significantly associated with mefloquine administration. Nausea and abdominal pain showed a similar tendency, not achieving statistical significance. It is possible that the dosing schedule, recommendations regarding pregnancy testing and contraception, and the symptoms listed in the diary suggested the identity of the active formulation to volunteers with medical knowledge and/or previous experience of mefloquine through travel. Increased rates and ratings of self-reported neuropsychiatric symptoms, 


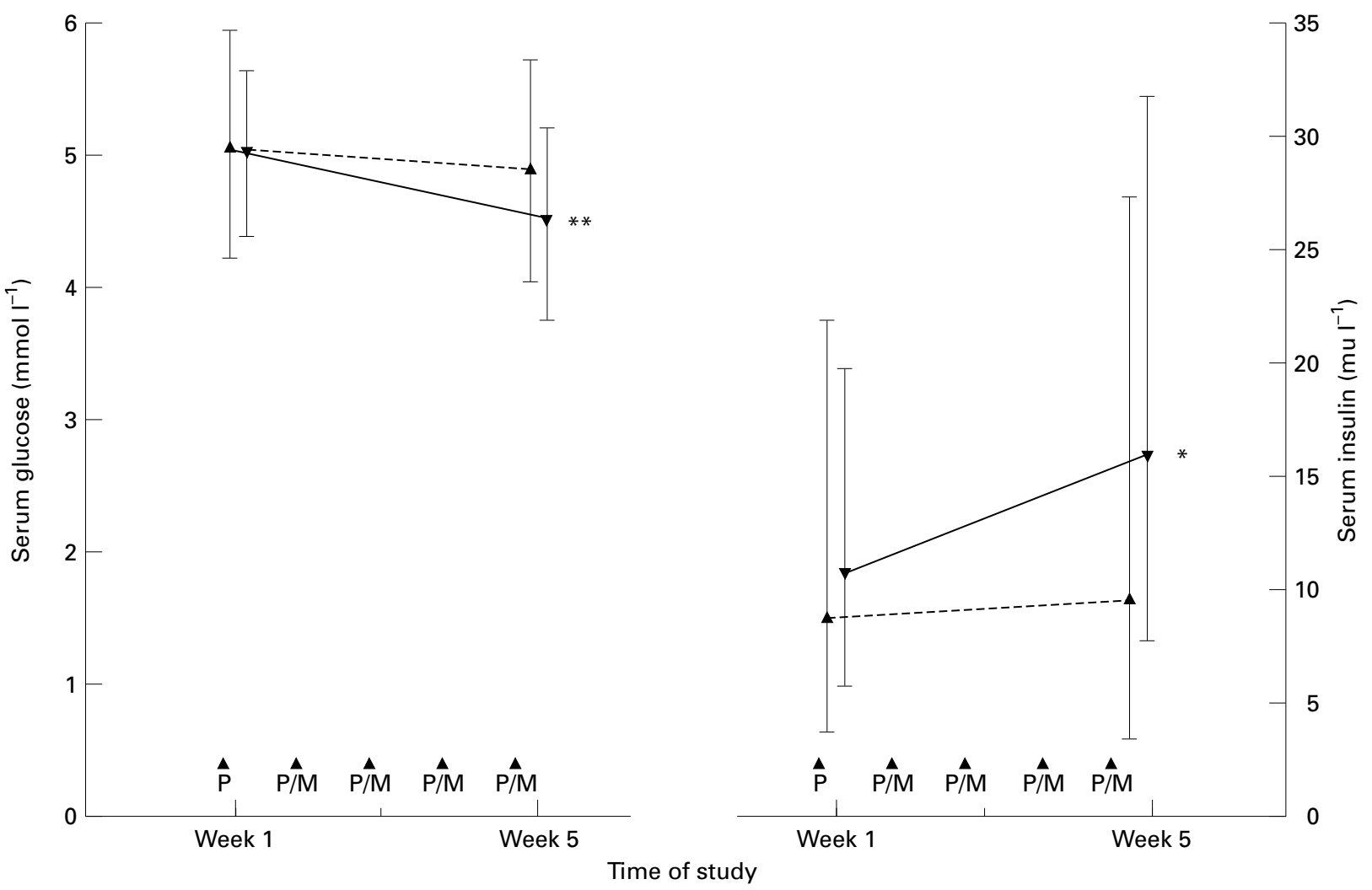

Figure 2 Serum glucose (mean \pm s.d.; left panel) and insulin (geometric mean \pm s.d.; right panel) in patients allocated mefloquine $(\boldsymbol{\nabla}-\boldsymbol{\nabla})$ before (week 1) and at the end of (week 5) four weekly doses of $250 \mathrm{mg}$, and in the placebo group ( $\mathbf{\Delta}---\boldsymbol{\Lambda})$ at the same times. There was a significant fall in serum glucose $(* * P<0.001)$ and rise in insulin $(* P<0.01)$ in the mefloquine group.

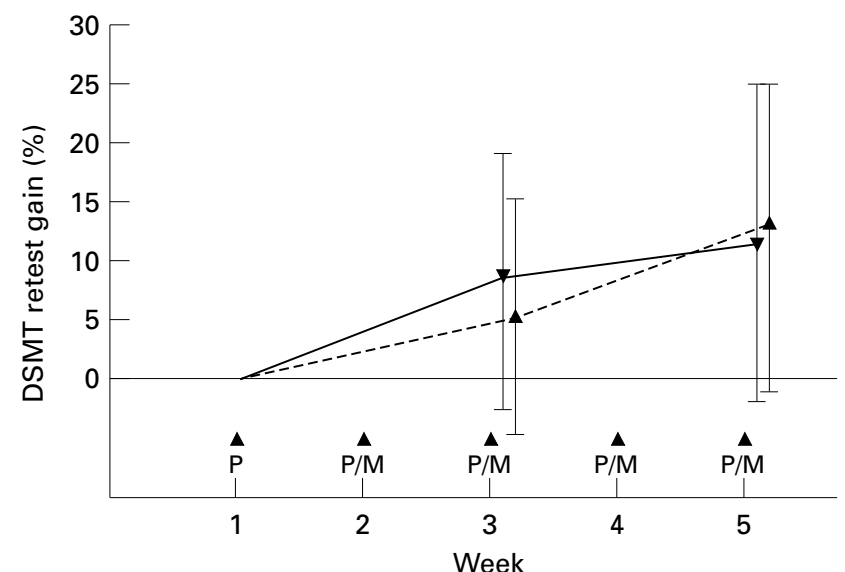

Figure 3 Retest gain as a percentage of baseline for Digit Symbol Modalities Test score in subjects receiving mefloquine $(\boldsymbol{\nabla}-\boldsymbol{\nabla})$ or placebo $(\boldsymbol{\Delta}--\boldsymbol{\Delta})$ over four weeks.

and a possible bias against mefloquine, might have been expected in this situation. Given our results and those of other trials involving young volunteers [18], there was little evidence of such an effect in the present study.

In other large-scale studies, nausea, dizziness and headaches were reported by between 6 and $12 \%$ of European travellers on mefloquine [2], while strange dreams, insomnia and nausea were present in 10 to $25 \%$ of U.S. Peace Corps volunteers on long-term prophylaxis [3]. These data suggest that different sideeffect profiles reflect discrepancies between demographic characteristics of study populations, as well as itinerary, comedication rates and even pre-travel advice and warnings. Nevertheless, gastrointestinal side-effects seem
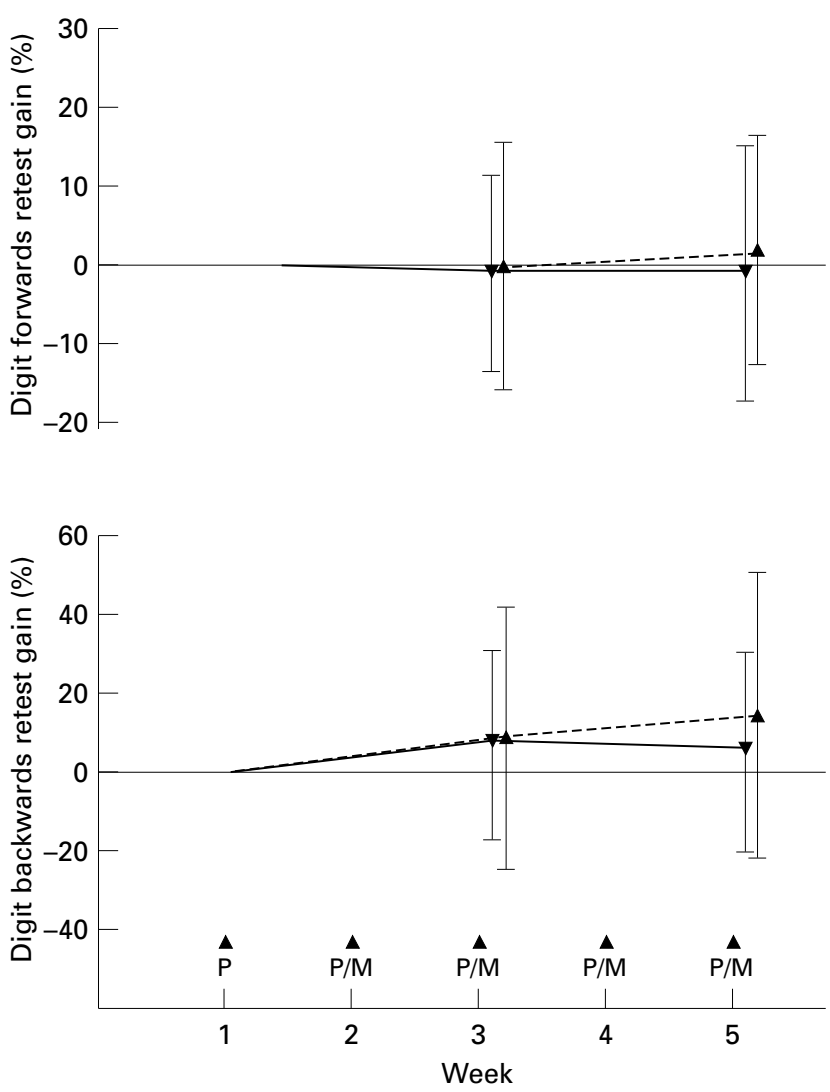

Figure 4 Retest gain as a percentage of baseline for digit span forwards (top panel) and digit span backwards (bottom panel) test scores in subjects receiving mefloquine $(\boldsymbol{\nabla}-\boldsymbol{\nabla})$ or placebo ( $\boldsymbol{\Lambda}---\boldsymbol{\Delta})$ over four weeks. 
Table 2 Auditory threshold, systolic (SBP) and diastolic (DBP) blood pressure, and $\mathrm{QT}_{\mathrm{c}}$ interval changes in the two groups. First assessment data are mean \pm s.d. except for auditory threshold which are median and (interquartile range) for the average of right and left ears. The mean difference from the first assessment and (95\% confidence intervals) are listed for the second and third assessments.

\begin{tabular}{lccc}
\hline & First assessment & Second assessment & Third assessment \\
\hline Mefloquine group $(n=46)$ & & & $0(-2.0,2.0)$ \\
Hearing loss at 6 kHz $(\mathrm{dB})$ & $17.5(12.0-22.5)$ & $-2.5(0,4.5)$ & $0(-3,3)$ \\
Fall in SBP on standing $(\mathrm{mm} \mathrm{Hg})$ & $-1 \pm 9$ & $-4(-7,0)$ & $1(-2,4)$ \\
Fall in DBP on standing $(\mathrm{mm} \mathrm{Hg})$ & $-10 \pm 6$ & $2(-1,5)$ & $3(-2,8)$ \\
QT $_{\mathrm{c}}$ interval $(\mathrm{ms})$ & $404 \pm 23$ & $10(4,16)^{* *}$ & \\
& & & $-1.5(-3.5,0.5)$ \\
Placebo group $(n=49)$ & $17.5(13.5-25.0)$ & $-1.5(-4.0,0.5)$ & $3(0,6)$ \\
Hearing loss at $6 \mathrm{kHz}(\mathrm{dB})$ & $-3 \pm 8$ & $-5(0,10)$ & $2(0,4)$ \\
Fall in SBP on standing $(\mathrm{mm} \mathrm{Hg})$ & $-13 \pm 6$ & $1(-1,3)$ & $15(1,29)^{*}$ \\
Fall in DBP on standing $(\mathrm{mm} \mathrm{Hg})$ & $400 \pm 49$ & $10(-2,22)$ & \\
QT interval $(\mathrm{ms})$ & & & \\
\hline
\end{tabular}

$* P<0.05 ; * * P<0.01$

to be the most consistent in all studies to date. Our data do not support an increase in mild and debilitating neurological symptoms during mefloquine prophylaxis. Consistent with known frequency of serious neuropsychiatric reactions from large scale trials $[10,11]$, none was observed in our relatively small sample.

The change in serum albumin over the study period in both groups is difficult to explain but of limited biological significance. The stability of serum ionised calcium and magnesium during weekly mefloquine dosing argues against disturbances in mineral homeostasis contributing to even mild neurological effects. The fall in plasma glucose seen in subjects taking mefloquine was proportionately greater than that in serum albumin and appeared to result from relative hyperinsulinaemia. Mefloquine is related chemically to the cinchona alkaloids. It is, therefore, possible that it has a quininelike effect on the pancreatic beta-cell [24] even though the correlation between plasma drug concentrations and changes in serum insulin from baseline did not achieve statistical significance.

There are limited data on the effect of mefloquine on glucose homeostasis. In a study involving seven healthy volunteers, plasma glucose and insulin remained close to baseline during the $6 \mathrm{~h}$ after a single dose [25], a period which is much shorter than the time at which maximal plasma mefloquine concentrations are usually reached [23]. Although our subjects were not studied in a fasting or timed post-prandial state, most attended several hours after eating. Serum glucose and insulin concentrations were largely within post-absorptive ranges and suggest that peak mefloquine concentrations on a background of regular dosing may result in hyperinsulinaemia. This effect, when coupled with other factors such as prolonged fasting or ethanol ingestion, could produce hypoglycaemic symptoms and even serious consequences of neuroglycopaenia such as convulsions [12]. Recent reports of mefloquineassociated hypoglycaemia in a cachectic patient with AIDS [26] and in an individual after alcohol consumption [27] support this hypothesis.

Psychometric testing revealed no significant effects of mefloquine on neurological performance. This reflects both the infrequent reporting of impairment of memory, concentration and motor function by those volunteers on active formulation and previous data from young subjects on long-term mefloquine [3] in whom less than $2 \%$ considered that the drug interfered with daily activities. Median values for $6 \mathrm{kHz}$ auditory threshold in our subject groups at baseline were similar to that in a recent survey of young adults who were not prescreened for otological abnormalities (15.5 dB [28]). Despite evidence of high-tone auditory toxicity of quinine in healthy subjects [29], we found no increase in hearing loss due to mefloquine though, due to the testing step used, low-grade $(<5 \mathrm{~dB})$ changes may have been missed. Postural blood pressure responses were unaffected by mefloquine. There was, however, transient if mild $\mathrm{QT}_{\mathrm{c}}$ prolongation which, given that there is some evidence that the interaction between mefloquine and halofantrine may predispose to serious cardiac arrhythmias [30], suggests that care should be exercised when mefloquine is prescribed with other drugs known to influence ventricular repolarisation.

The present study adds to available data confirming the relative safety of prophylactic doses of mefloquine in healthy subjects. There was no clear subjective or objective evidence of mefloquine-associated neurological dysfunction or cardiovascular toxicity apart from mild and transient $\mathrm{QT}_{\mathrm{c}}$ prolongation. Gastrointestinal sideeffects were relatively common, especially diarrhoea, but these symptoms were also mild and short-lived. Significant biochemical changes in subjects on active formulation were restricted to an average $0.5 \mathrm{mmol}^{-1}$ reduction in plasma glucose; under certain circumstances, mefloquine-associated hyperinsulinaemia might have more serious consequences [12, 26, 27]. Although mental stimulation in the form of overseas travel could be an important substrate for subtle mefloquineassociated neurotoxicity, it is possible that information travellers obtain relating to potential problems of antimalarial prophylaxis may also contribute to subsequent symptomatology.

We thank Janet Allen for help with organisation, testing and data handling, Andrew St John, Ken Robertson and Wayne 
Johnston for biochemical assays, Yngve Bergqvist for drug and metabolite assays, Caroliene Giele, Bruce Maley and Gary Harbord for assistance with clinical testing, and F. Hoffmann La Roche \& Co. for financial support.

\section{References}

1 Boudreau E, Schuster B, Sanchez J, et al. Tolerability of prophylactic Lariam regimens. Trop Med Parasitol 1993; 44: $257-265$.

2 Steffen R, Fuchs E, Schildknecht J, et al.. Mefloquine compared with other chemoprophylactic regimens in tourists visiting east Africa. Lancet 1993; 341: 1299-1303.

3 Lobel HO, Miani M, Eng T, Bernard KW, Hightower AW, Campbell CC. Long term malaria prophylaxis with weekly mefloquine. Lancet 1993; 341: 848-851.

4 Van den Enden E, Van Gompel A, Colebunders R, van den Enden J. Mefloquine-induced Stevens-Johnson syndrome. Lancet 1991; 337: 683.

5 Martin GJ, Malone JL, Ross EV. Exfoliative dermatitis during malarial prophylaxis with mefloquine. Clin Infect Dis 1993; 16: 341-342.

6 Scerri L, Pace JL. Mefloquine-associated cutaneous vasculitis. Int J Dermatol 1993; 32: 517-518.

7 Hennequin C, Bouree P, Halfon P. Agranulocytosis during treatment with mefloquine. Lancet 1991; 337: 984.

8 Stracher AR, Stoeckle MY, Giordano MF. Aplastic anaemia during malarial prophylaxis with mefloquine. Clin Infect Dis 1994; 18: 263-264.

9 World Health Organisation. Prophylactic and therapeutic use of mefloquine. Weekly Epidemiologic Record 1989; 32: 247-248.

10 Weinke T, Trautmann M, Held T, et al. Neuropsychiatric side-effects after the use of mefloquine. Am J Trop Med Hyg 1991; 45: 86-91.

11 Bjorkman A. Acute psychosis following mefloquine prophylaxis. Lancet 1989, ii: 865.

12 Bem JL, Kerr L, Sturchler D. Mefloquine prophylaxis: an overview of spontaneous reports of severe psychiatric reactions and convulsions. J Trop Med Hyg 1992; 95: 167-179.

13 Patchen LC, Campbell CC, Williams SB. Neurologic reactions after a therapeutic dose of mefloquine. $N \mathrm{Engl}$ $J$ Med 1989; 321: 1415-1416.

14 Lapras J, Vighetto A, Trillet M, Garin JP. Trouble transitoire de memoire au decours d'un acces palustre. Presse Med 1989; 18: 776.

15 Caillon E, Schmitt L, Moron P. Acute depressive symptoms after mefloquine treatment. Am J Psychiatry 1992; 149: 712.
16 Olson PE, Kennedy CA, Morte PD. Paresthesias and mefloquine prophylaxis. Ann Int Med 1992; 117: 1058-1059.

17 Rombo L, Angel VH, Friman G, Hellgren U, Mittelholzer ML, Sturchler D. Comparative tolerability and kinetics during long-term intake of Larium and Fansidar for malaria prophylaxis in nonimmune volunteers. Trop Med Parasitol 1993; 44: 254-256.

18 Rosenzweig P, Brohier S, Zipfel A. The placebo effect in healthy volunteers: influence of experimental conditions on the adverse events profile during phase I studies. Clin Pharmacol Ther 1993; 54: 578-583.

19 Smith A. Symbol digit modalities test manual. Western Psychological Services 1973.

20 Wechsler D. Manual for the Wechsler adult intelligence scale. New York, The Psychological Corporation 1955: 44.

21 Bergqvist Y, Hellgren U, Churchill F. High-performance liquid chromatography assay for the simultaneous monitoring of mefloquine and its acid metabolite in biological samples using protein precipitation and ion-pair extraction. J Chromatogr 1988; 432: 253-263.

22 Pennie RA, Koren G, Crevoisier C. Steady state pharmacokinetics of mefloquine in long-term travellers. Trans $R$ Soc Trop Med Hyg 1993; 87: 459-462.

23 Mimica I, Fry W, Eckert G, Schwarz DE. Multiple dose kinetic study of mefloquine in healthy male volunteers. Chemotherapy 1983; 29: 184-187.

24 Davis TME, Karbwang J, Looareesuwan S, Turner RC, White NJ. Comparative effects of quinine and quinidine on glucose metabolism in normal man. Br J Clin Pharmacol 1990; 30: 397-403.

25 Phillips RE, Looareesuwan S, White NJ, et al. Hypoglycaemia and antimalarial drugs: quinidine and release of insulin. Br Med $J$ 1986; 292: 1319-1321.

26 Assan R, Perronne C, Chotard L, Larger E, Vilde JL. Mefloquine-associated hypoglycaemia in a cachectic AIDS patient. Diabete Metab 1995; 21: 54-58.

27 Wittes R, Sanigur R. Adverse reaction to mefloquine associated with ethanol ingestion. Can Med Ass J 1995; 152: 515-517.

28 Lutman ME, Davis AC. The distribution of hearing threshold levels in the general population aged 18-30 years. Audiology 1994; 33: 327-350.

29 Roche RJ, Silamut K, Pukrittayakamee S, et al. Quinine induces reversible high-tone hearing loss. $\mathrm{Br} J$ Clin Pharmacol 1990; 29: 780-782.

30 Nosten F, Ter Kuile FO, Luxemberger C, et al. Cardiac effects of antimalarial treatment with halofantrine. Lancet 1993; 341: 1054-1056.

(Received 13 March 1996, accepted 2 July 1996) 